von mir hervorgehobene Identität $\mathrm{zu}$ erschüttern, und meine Bemerkung erscheint deshalb durchaus nicht so "bedeutungslos", wie Herr Gruenhagen auf Grund seines Nichts beweisenden Versuchs bewiesen zu haben glaubt.

Um diesen Brief mit etwas Erfreulicherem zu schliessen, gestatten Sie mir wohl noch eine Bemerkung zu der Arbeit von Goltz über die Functionen des Lendenmarks. Herr Goltz berichtet über die "überaus auffällige Thatsache, dass die Durchtrennung eines Nerven auch dann noch Temperatursteigerung an der Peripherie vermitteln kann, wenn dieser Nerv keinen Zusammenhang hat mit den bestehenden Centren". Herr Goltz hat übersehen, dass die von ihm beobachtete Thatsache schon mitgetheilt und vollkommen ausreichend erklärt ist von Claude Bernard. (S. Comptes rendus etc. LV, 228 und mein Referat darüber im Centralbl. f. d. med. Wissensch. 1863, S. 20.)

Sie würden mich sehr verbinden, wenn Sie diesen Zeilen einen Raum in Ihrem Archiv gönnen wollten. Ich halte mich gern von jeder Polemik fern und habe seit Jahren Vieles schweigend hingenommen, was vielleicht Erwiderung verdient hätte. Herrn Gruenhagen gegenüber glaubte ich diesmal nicht schweigen zu dürfen. Mit der Versicherung freundschaftlicher Hochachtung der Ihrige

I. Rosenthal.

\title{
Das Knochenmark als Organ der Blutbildung.
}

Eine Erwiderung an Mr. Charles Robin

von

Professor E. Neumann in Königsberg i. Pr.

Herr Robin hat in dem diesjährigen ersten Hefte des von ihm herausgegebenen Journal de l'anatomie et de la physiologie normales et pathologiques einen längeren Aufsatz "sur la moelle des os " veröffentlicht, in welchem derselbe die diesem Organe von mir zugeschriebene wichtige physiologische Function, der Blutbildung zu dienen, nicht nur in Frage stellt, sondern geradezu leugnet. Ich glaube, diese negirenden Behauptungen im Interesse der Sache nicht ohne einige Worte der Erwiderung lassen zu dürfen, 
da ich Grund habe anzunehmen, dass bisher nur wenige Forscher durch eigene Untersuchungen sich ein Urtheil in dieser Frage gebildet haben und dass daher Mancher geneigt sein dürfte, den Ausführungen des Herrn Robin einen Werth beizumessen, der ihnen wahrlich nicht zukommt.

Obwohl Herr Robin meine im zehnten Bande des Archivs für Heilkunde 1868 erschienene Abhandlung müber die Bedeutung des Knochenmarkes für die Blutbildung “ am angeführten Orte citirt, so muss ich doch glauben, dass ihn entweder die Lektüre derselben nur in sehr unvollkommener Weise mit ihrem Inhalte vertraut gemacht oder dass er sich mit einem dürftigen und sinnentstellenden französischen Referate begnügt hat. Nur so ist es verständlich, dass Herrn Robin's Aufsatz dasjenige Factum, welches ich als das Fundament meiner Argumentation hingestellt habe und welches ich bereits in einer vorläufigen Mittheilung (Centralbl. f. d. med. Wiss. 1868, Nr. 44) annoncirt hatte, mit völligem Stillschweigen übergeht. Dieses Factum ist das auf dem von mir angegebenen Wege (Ausbreitung des aus dem Knochen ausgequetschten Marksaftes in dünnster Schicht $\mathrm{ohne}$ jeden $\mathrm{Zus}$ atz von Flüssigkeiten) jederzeit im rothen Knochenmarke leicht nachweisbare Vorkommen kernhaltiger rother Blutzellen, welche mit den kernhaltigen rothen Blutzellen des Embryo völlig übereinstimmen ${ }^{1}$ ).

Wollte Herr Robin demnach meine Behauptung, dass im rothen Knochenmark fortdauernd eine Entwicklung farbiger Blutzellen stattfindet, widerlegen, so war es seine Aufgabe entweder zu beweisen, dass in demselben Zellen, wie ich sie beschrieben, nicht vorkommen, od er, falls er meine Beschreibung derselben bestätigt fand, zu zeigen, dass man sie trotzdem mit den kernhaltigen rothen Blutzellen des Embryo nicht identificiren dürfe, od er endlich, falls sich auch diese Identität nicht leugnen liess, darzuthun, dass es ganz irrig ist, wie allgemein geschieht, die kernhaltigen Blutkörper des Embryo als Entwicklungsstufen der farbigen kernlosen Blutkörper zu betrachten.

Herr R obin hat sich, wie gesagt, die Sache leichter gemacht. Es scheint ihm völlig gleichgültig zu sein, dass ich Elemente der genannten Qualität im Knochenmark aufgefunden und beschrieben

1) Als vorzüglich geeignetes und stets leicht zu beschaffendes Untersuchungsobject empfehle ich hier nochmals die Rippen menschlicher Leichen. 
habe und dass Herr Bizzozero u. A. diesen Fund alsbald bestätigten; es lag für ihn daher auch keine Veranlassung vor, nach denselben zu suchen, am wenigsten, dabei nach der von mir empfohlenen Untersuchungsmethode zu verfahren.

Wenn wir somit sehen, dass sich Herr Robin mit den fü $r$ die Entwicklung von Blutzellen im Marke angeführten Beobachtungen einfach dadurch abfindet, dass er sie ignorirt, und dass er nicht einmal den Versuch macht, sie zu widerlegen, so liesse sich wenigstens erwarten, dass er seinerseits Thatsachen ins Feld führt, welche als Beweise gegen einen solchen Vorgang gelten können. Es würde alsdann Aufgabe der weiteren Forschung sein, die Gründe pro und contra abzuwägen. Auch hierin wird unsere Erwartung gänzlich getäuscht. Was er vorbringt reducirt sich auf Folgendes:

1. Ein Hauptaccent wird darauf gelegt, dass angeblich die Zellen des Markgewebes (médullocelles) und die farblosen Blutzellen (leucocythes) von einander durchaus verschieden sind, dass es demnach unstatthaft ist, anzunehmen, dass erstere durch Uebertritt in die Gefässe zu farblosen Blutzellen werden und sich als solche weiter entwickeln. Unbegreiflicher Weise sagt Herr Robin, dass vom Standpunkte der Logik aus eine Uebereinstimmung zwischen Markzellen und farblosen Blutzellen das erste Erforderniss für die blutbildende Function des Markes sei. Er übersieht dabei gänzlich, dass die Frage, o b im Marke Blutzellen zur Entwicklung gelangen, zu trennen ist von der Frage, woher die sich entwickelnden Blutzellen stammen? Liefern die Markzellen nicht das Material zu denselben, so bleibt ja noch die Möglichkeit, dass die Entwicklung stattfindet in den farblosen Blutzellen, welche dem Knochenmarke durch den arteriellen Blutstrom fortdauernd zugeführt werden. Sicher hätte Herr Robin nicht so viel Mühe verschwendet, um den Unterschied zwischen médullocelles und leucocythes nachzuweisen, wenn es ihm gegenwärtig gewesen wäre, dass ich trotz der positiven Behauptung, dass sich rothe Blutzellen im Marke bilden, die "Einwanderung von Markzellen in die Gefässe" nur als wahrscheinlich, aber keineswegs als conditio sine qua non hingestellt habe. Prüfen wir indessen, wie weit Herrn Robin der Nachweis geglückt ist, dass beide Zellarten so absolut verschieden sind.

Ich behaupte, dass seine Angaben nur lehren, was noch Niemand, soviel mir bekannt, bezweifelt hat, dass in dem Knochenmarke zahlreiche Zellen vorkommen, die in gleicher Qualität im Blut nicht 
vorkommen, dass aber ein Beweis dafür, dass sämmtlich e Zellen des Markgewebes von den farblosen Blutzellen verschieden sind, nicht geführt ist und nicht geführt werden konnte. Was lag denn Herrn Robin zur Vergleichung vor? jedenfalls nicht Blutzellen auf der einen und Markzellen auf der andern Seite, sondern sein Vergleich musste sich beschränken auf das Blut und eine Mischung von Blut mit Mark, denn es ist selbstverständlich nicht angänglich, bei der Herstellung der Präparate eine Zumischung des Inhaltes der Blutgefässe zu dem Markgewebe zu verhüten. Wie konnte nun aber Herr Robin in einer solchen Mischung erkennen, $o b$ eine in derselben vorhandene, ausserhalb eines Gefässes befindliche Zelle von der Qualität eines farblosen Blutkörperchens bei der Präparation einem Blutgefässe entschlüpft war oder ob sie sich ursprünglich bereits ausserhalb des Blutgefässes im Markgewebe befand? Wie war es also möglich, dass er die von ihm mit so vieler Bestimmtheit ausgesprochene Ueberzeugung gewann, dass Zellen von der Beschaffenheit der farblosen Blutzellen im Markgewebe nicht vorkommen? Es wäre zur Begründung eines solchen Ausspruches unbedingt erforderlich gewesen, das Mark nicht nur im frischen weichen Zustande, sondern auch nach vorheriger Erhärtung zu untersuchen, um die zelligen Elemente im Innern der Blutgefässe und diejenigen des Markes in situ an mikroskopischen Schnittpräparaten sehen zu können; so lange nicht eine die Zellen in ihrer natürlichen Beschaffenheit conservirende Erhärtungsmethode bekannt ist, wird eine sichere Entscheidung darïber nicht zu erreichen sein.

2. Als ein zweites Argument macht Herr Robin geltend, dass gewisse als blutkörperchenhaltige Zellen beschriebene Elemente des Markes "cellules qui ont été dites hématopoétiques par quełques auteurs« (?) seiner Beobachtung zufolge nicht wirklich Blutkörperchen, sondern vielmehr Pigmentkörner von der Farbe der Blutkörper (goûttes d'hématosine) einschliessen, wie sich aus der Résistenz dieser Körper gegen Wasser und Essigsäure ergiebt. Abgesehen davon, däss es nicht schwierig ist, sich nach der von mir benutzten Untersuchungsmethode davon zu überzeugen, dass neben Zellen mit Pigmentmassen auch wirkliche blutkörperchenhaltige Zellen im Knochenmarke unter gewissen Umständen vorkommen, Zellen, bei denen man durch Zusatz von Wasser oder Essigsäure in der That eine Zerstörung der Inhaltskörper erreicht, abgesehen sage ich von dieser Lücke in den Beobachtungen Robin's, muss ich bemerken, 
dass diese blutkörperchenhaltigen Zellen mit der uns beschäftigenden Frage gar Nichts zu thun haben. Herr Robin scheint bei ihrer Erwähnung Angaben aus früheren Zeiten im Sinne gehabt zu haben, wo die Auffindung blutkörperchenhaltiger Zellen in der Milz (deren Existenz von ihm gleichfalls irrthümlicher Weise in Abrede gestellt wird) allerdings einigen Forschern Veranlassung gab, dieselben so zu deuten, als fände hier eine Neubildung von Blutkörpern im Innern von Zelleu statt. Die blutkörperchenhaltigen Zellen des Knochenmarkes sind weder von mir, noch, soviel mir bekannt, von irgend einer andereu Seite in diesem Sinne verwerthet worden, im Gegentheil hat Bizzozer o (meiner Ansicht nach allerdings mit Unrecht) aus ihrem Vorkommen den Schluss gezogen, dass im Knochenmark beständig eine gewisse Zahl von Blutkörpern ihren Untergang fände.

3. Mit nicht grösserem Glücke, wie mir scheint, appellirt Herr Robin endlich noch an einige andere Thatsachen. Er findet es mit der Annahme einer Blutbildung im rothen Knochenmarke nicht verträglich, dass dasselbe im Laufe der Körperentwicklung grossentheils aus den Knochen verschwindet und durch gelbes Fettmark ersetzt wird, dass bei den Vögeln ein grosser Theil der Knochen gar kein Mark enthält, sondern von den Luftsäcken ausgefüllt wird, dass ferner die Knorpelfische überhaupt gar kein rothes Mark besitzen. Ich möchte meinen, dass man mit derselben Logik berechtigt wäre, zu bezweifeln, dass die Lungen der Respiration dienen, weil der Fötus sich seiner Lungen nicht zum Athmen bedient oder weil es Thiere giebt, welche athmen, ohne im Besitz von Lungen zu sein.

Es bedarf wohl kaum eines weiteren Beweises, um zu zeigen, dass der von Herrn Ro bin erhobene Vorwurf non demeure confondu du peu de rigneur, que mettent dans leurs observations beaucoup de ceux qui viennent ainsi encombrer la science " auf ihn selbst zurückfällt und dass sein absprechendes Urtheil sich auf ein durchaus ungenügendes Beobachtungsmaterial und ein durchaus fehlerhaftes Raisonnement gründet. Ich gebe mich daher der Hoffnung hin, dass trotz Herrn Robin's Widerspruch die Entwicklung von Blutkörpern im Knochenmark in nicht langer Zeit allgemein als eine feststehende und leicht constatirbare physiologische Thatsache anerkannt sein wird.

Schliesslich noch einige Worte über einen Prioritätsanspruch des Herrn Robin mir gegenüber. Wie er in Erinnerung bringt, 
hatte er bereits im Jahre 1864 als Eigenthümlichkeiten der Markcapillaren ihre grössere Weite im Vergleich zu den Capillaren des Periost und des Knochens und ihre sinuöse Form erwähnt. Ich meinerseits habe, ohne diese Angaben Robin's zu kennen, die Weite der Markcapillaren gegenüber den 'Arterien und das Vorkommen "divertikelartiger Ausstülpungen der Capillarwand in Form von zugespitzten und meistens in einen feinen Faden auslaufenden Sprossen, entsprechend den Gefässanlagen im Schwanze der Froschlarven " hervorgehoben. Dass diese Beobachtungen eine einfache Bestätigung dessen, was Herr Robin beschrieben, darstellen, wie dieser behauptet, kann ich nicht zugeben.

Königsberg, den 31. März 1874 .

Ueber

die Wirkungen des Kaffees, des Fleischextractes und der Kalisalze auf Herzthätigkeit und Blutdruck.

Von

H. Aubert und A. Dehn in Rostock.

In einer früheren Arbeit über die Wirkungen des Coffeins war A ubert zu dem Resultate gekommen, dass im Kaffeegetränk ausser dem Coffein noch ein anderer energisch virkender Stoff enthalten sein müsste, da Thiere, welchen der coffeinfreie Rückstand des Kaffeefiltrates injicirt wurde, rasch und zwar mittelst Herztodes starben. Er vermuthete, dass das im Kaffee enthaltene Kali diese Wirkung hervorbrächte und fand zunächst eine Bestätigung dieser Vermuthung in der Thatsache, dass die Injection einer Lösung des geglühten und veraschten Kaffeefiltratrückstandes eben dieselbe Wirkung hervorbrachte, wie das Kaffeefiltrat. Diese Erfahrung veranlasste uns, eine Reihe von Versuchen über die Wirkungen verschiedener Kalisalze im Vergleich zu den Wirkungen des kalihaltigen Kaffeefiltrates und kalihaltigen Fleischextractes anzustellen und zunächst den Einfluss dieser Substanzen auf die Herzthätigkeit zu prüfen. Unsere Versuche haben dargethan, dass diese Substanzen wesentlich durch ihren Gehalt an Kali auf das Herz wirken.

Um vergleichbare Versuche anstellen zu können, musste der Kaligehalt des Kaffeefiltrates festgestellt, und sowohl für diese Sub- 